\title{
Pre-existing intratumoral CD8 T cells substantially contribute to control tumors following therapeutic anti-CD40 and polyl:C based vaccination
}

\author{
Aaron D. Stevens ${ }^{1}$ and Timothy N.J. Bullock ${ }^{1}$ \\ ${ }^{1}$ Department of Pathology, University of Virginia, Charlottesville, VA, USA
}

Corresponding author: Timothy N.J. Bullock; Carter Harrison Bldg Rm G527, 345 Crispell Dr, Charlottesville, VA, USA, 22908; tb5v@virginia.edu

Word count: 4972

Keywords: Vaccination, CD40, TLR3, CD8 T cells, Trafficking, Cancer immunotherapy, Melanoma

Author Contributions: ADS and TNJB conceived the project, designed experiments, interpreted data, and wrote the manuscript. ADS performed experiments and analyzed data. TNJB supervised and acquired funding.

Funding: This work was funded by National Institutes of Health grant R01CA166458 to TNJB. ADS was supported by National Institutes of Health training grant T32AI007496.

Ethics approval: All mice were treated in accordance with policies established by the University of Virginia Animal Care and Use Committee (protocol 3292).

Data availability statement: All data relevant to the study are included in the article or uploaded as online supplementary information. Additional information regarding data may be obtained

19 from the authors on reasonable request. 
bioRxiv preprint doi: https://doi.org/10.1101/2020.08.31.275420; this version posted September 1, 2020. The copyright holder for this preprint (which was not certified by peer review) is the author/funder. All rights reserved. No reuse allowed without permission.

Pre-existing intratumoral CD8 T cells substantially contribute to control tumors following therapeutic anti-CD40 and polyl:C based vaccination

20 Abbreviations: dendritic cells (DC), polyinosinic-polycytidylic acid (polyl:C), toll like receptor

21 (TLR), brefeldin A (BFA) 
Pre-existing intratumoral CD8 T cells substantially contribute to control tumors following therapeutic anti-CD40 and polyl:C based vaccination

\section{ABSTRACT}

Background Dendritic cells are potently activated by the synergistic action of CD40 stimulation

in conjunction with signaling through toll like receptors, subsequently activating antigen

specific T cells. Cancer vaccines targeting the activation of dendritic cells in this manner show

promise in murine models and are being developed for human cancer patients. While vaccine

efficacy has been established, further investigation is needed to understand the mechanism of

tumor control and how vaccination alters tumor infiltrating immune cells.

Methods Mice bearing established murine melanoma tumors were vaccinated with agonist

anti-CD40, polyl:C, and tumor antigen. Intratumoral T cell numbers, differentiation state, to examine changes to intratumoral T cells present at the time of vaccination.

Results Vaccination led to increased intratumoral T cell numbers and delayed tumor growth.

Expansion of T cells and tumor control did not require trafficking of T cells from the periphery.

The increase in intratumoral T cells was associated with an acute burst in proliferation but not numbers yielded increased effector T cells per tumor. 
Pre-existing intratumoral CD8 T cells substantially contribute to control tumors following therapeutic anti-CD40 and polyl:C based vaccination

42 This indicates that the existing T cell response and intratumoral DC could be critical for

43 vaccination efficacy. This also suggests that circulating T cells may not be an appropriate

44 biomarker for vaccination efficacy.

\section{BACKGROUND}

Recent advances in checkpoint blockade and adoptive cell therapy demonstrate the potential

47 for using adaptive immunity to fight cancer. Immune infiltration of tumors, especially by cytotoxic CD8 T cells, correlates with improved patient prognosis overall and in response to anti-PD1 therapy.(1,2) Therefore, boosting the presence of CD8 T cells in tumors is a major goal

50 of cancer immunotherapy. One approach to induce CD8 T cell expansion in cancer patients is

51 through vaccination. Early human vaccination trials were generally unsuccessful at controlling

52 tumor growth, despite expanding antigen specific T cells.(3-7) More recent trials expanding the range of adjuvants or combining vaccination with other immunotherapies have improved

54 the number of patients with stable or regressing tumors, but the magnitude of induced T cell expansion remains far below what is seen in response to infection in humans or with vaccination in tumor bearing mice.(8-11) As human vaccine trials evolve to incorporate next

57 generation adjuvants and neoantigens, preclinical studies are needed to investigate the best combination of adjuvants for cancer vaccines and characterize their synergistic effects on immune responses. In vivo, interactions with mature antigen presenting cells, primarily dendritic cells (DC), lead to

61 CD8 T cell activation. One of the key drivers of DC maturation and subsequent T cell activation

62 is stimulation of CD40 on DC, through engagement with CD40L on CD4 T cells.(12) While toll 
Pre-existing intratumoral CD8 T cells substantially contribute to control tumors following therapeutic anti-CD40 and polyl:C based vaccination

63 like receptor (TLR) agonists show some ability to promote T cell responses to

64 vaccination, $(13,14)$ the combination of CD40 stimulation and TLR agonists synergize to drive

65 robust antigen specific CD8 T cell expansion.(15) The combination of $\alpha \mathrm{CD} 40$ and TLR agonists

66 has been shown to drive CD8 T cell expansion through upregulation of CD70 on $D C(16,17)$ and

67 to be dependent on IFN $\alpha / \beta .(11,15)$ Supporting the notion that activating DC promotes anti-

tumor immunity, several studies demonstrate that vaccine approaches combining $\alpha \mathrm{CD} 40$

agonistic antibody with TLR agonists are effective in mouse models of cancer.(11,18-21)

Despite the efficacy of vaccination with $\alpha \mathrm{CD} 40$, polyinosinic-polycytidylic acid (polyl:C) and

71 antigen in murine tumor models, the mechanistic basis for tumor control is still unclear. Some

72 studies suggest that CD40-mediated activation of myeloid cells is sufficient for tumor

control,(22-24) while others demonstrate that $\alpha \mathrm{CD} 40$ vaccination increases antigen specific

74 CD8 T cell numbers in the spleen and blood, and tumor control is dependent on CD8 T

75 cells. $(11,25)$ The contribution of CD4 T cells to tumor control after vaccination with $\alpha \mathrm{CD} 40$,

polyl:C, and antigens containing MHC class II epitopes is relatively unexplored. Whether

77 vaccination with $\alpha \mathrm{CD} 40$ and polyl:C alters the number and function of intratumoral T cells is

78 also unknown. Interestingly, while studies show that IFN $\gamma$ production by various effector cells

79 substantially contributes to tumor control in multiple model systems, tumor control with

$80 \alpha C D 40$, polyl:C, and peptide antigen vaccination is dependent on perforin but independent of

81 IFNy, suggesting that increased cytotoxicity may drive tumor control.(11) The subsets of

82 immune cells with improved anti-tumor cytotoxicity after vaccination with $\alpha \mathrm{CD} 40$ and polyl:C

83 have not been well characterized. Further, the relative contribution of pre-existing tumor 
Pre-existing intratumoral CD8 T cells substantially contribute to control tumors following therapeutic anti-CD40 and polyl:C based vaccination

84 infiltrating T cells as compared to newly primed T cells arriving from the periphery to vaccination induced tumor control is unclear.

Here, using a well-established murine model of melanoma, we have examined T cell responses

87 within the tumor after vaccination with $\alpha C D 40$, polyl:C, and tumor antigen. We show that either CD8 or CD4 T cells are sufficient for vaccination to delay tumor growth. We observe dramatically more CD8 and CD4 T cells in vaccinated tumors when trafficking is allowed and remarkably also when trafficking is blocked, indicating that vaccination promotes local expansion of T cells. Although vaccination expands T cells in the peripheral lymphoid tissues, we demonstrate that vaccination slows tumor growth without trafficking of additional T cells from the periphery. Both CD8 and CD4 T cells are more proliferative acutely after vaccination, although both $\mathrm{T}$ cell subsets do not maintain this increased proliferation and are ultimately not

95 better able to survive within the tumor. Surprisingly, CD8 T cells were acutely less functional in 96 situ after vaccination, although CD4 T cells were more functional after ex vivo stimulation. This

97 reduction in function occurred even when new T cells were allowed to flux into tumors.

98 However, the increased number of T cells counteracted their diminished function, ultimately

99 leading to equivalent or higher numbers of functional T cells in the tumors of vaccinated mice.

100 Therefore, vaccination limits tumor growth by sustaining the already infiltrated CD8 T cells

101 within the tumor, without requiring additional T cell infiltration.

\section{METHODS}

\section{Tumor model}


Pre-existing intratumoral CD8 T cells substantially contribute to control tumors following therapeutic anti-CD40 and polyl:C based vaccination

104 The B16cOVA tumor line was previously generated in our laboratory.(26) B16cOVA cells $\left(4 \times 10^{5}\right)$

105 were injected subcutaneously into the shoulder of C57BL/6 mice (National Cancer Institute).

106 Tumors were measured by calipers in two directions and reported as tumor area. Mice were

107 euthanized to harvest tumors at indicated time points or upon reaching the maximum allowed

108 size. All mice were treated in accordance with policies established by the University of Virginia

109 Animal Care and Use Committee.

\section{Treatments}

111 Tumor bearing mice were vaccinated i.p. with $100 \mu \mathrm{g} \alpha \mathrm{CD} 40$ (FGK45; BioXcell), $75 \mu \mathrm{g}$ polyl:C

112 (Invivogen), and $500 \mu \mathrm{g}$ ovalbumin (Sigma) or $200 \mu \mathrm{g} \mathrm{OVA}_{257-264}$ (Genscript) at day ten after

113 tumor injection. FTY720 (Sigma) was provided in the drinking water $(2 \mu \mathrm{g} / \mathrm{mL}$ ) starting at day

114 nine after tumor implantation and supplemented with daily i.p. injection $(25 \mu \mathrm{g})$ on days nine

115 through twelve. Depletion antibodies (250 $\mu \mathrm{g})$ for CD8 (2.43; BioXCell) and/or CD4 (GK1.5;

116 BioXCell) were administered i.p. at day eight after tumor injection and again every four days for

117 the remainder of the experiment. FTY720 and depletion efficiency were confirmed by flow

118 cytometric analysis of blood in each experiment. LFA blocking antibody (M17/4; BioXCell) was

119 administered i.p. (200 $\mu \mathrm{g})$ every two days. Brefeldin A (Thermo Fisher) was injected i.v. (250

$120 \mu \mathrm{g})$ five hours prior to harvest to block cytokine secretion. For OT1 transfer, spleens were

121 harvested from OT1 mice originally from Taconic and maintained at the University of Virginia.

\section{Sample preparation}

123 Tumors were excised and subjected to manual homogenization. T cells were isolated by density

124 gradient separation with Lympholyte-m (Cedarlane). For in vitro function, isolated T cells were 
Pre-existing intratumoral CD8 T cells substantially contribute to control tumors following therapeutic anti-CD40 and polyl:C based vaccination

125 stimulated with plate-bound $\alpha \mathrm{CD} 3(1 \mu \mathrm{g} / \mathrm{mL}$; eBioscience) or OVA 257 peptide pulsed, CD45-

126 mismatched splenocytes. H-2K

127 specific CD8. Antibodies and reagents used for staining are listed in table S1.

\section{Data analysis}

Flow samples were collected on Cytoflex S (Beckman Coulter) or Attune NxT (Thermo Fisher)

cytometers and analyzed using FlowJo V10. Statistical analyses were performed using

131 Graphpad Prism V8. Tumor outgrowth data is presented as mean \pm SEM from one

132 representative experiment and analyzed by Holm-Sidak multiple t tests. All other data is

133 presented as mean \pm SD and analyzed by Welch's $t$ test for two groups or Holm-Sidak multiple $t$

134 tests for more than two groups or time points. In some cases, pooled data is normalized to the

135 control samples within each experiment to compensate for batch effects and shown as the

136 relative expression of indicated factors. Differences were considered significant when $\mathrm{p}<0.05$.

\section{RESULTS}

138 Vaccination is effective in established murine melanoma

139 To assess the mechanism by which vaccination with a CD40 agonistic antibody, TLR3 agonist

140 polyl:C, and tumor antigen controls tumor growth, we utilized the B16cOVA melanoma model,

141 which allowed us to assess the immune response to the well-defined pseudo-neoantigen

142 ovalbumin (OVA) expressed by the tumor. When implanted subcutaneously, B16cOVA elicits a

143 modest inflammatory immune response, including both CD8 and CD4 T cells, that diminishes

144 over time and ultimately fails to control the tumor.(27) This provides the opportunity to 
Pre-existing intratumoral CD8 T cells substantially contribute to control tumors following therapeutic anti-CD40 and polyl:C based vaccination

145 improve both the function and number of infiltrating T cells. When tumor bearing mice were

146 vaccinated three and ten days after tumor implantation by i.p. injection of either whole OVA

147 protein or the CD8 immunodominant peptide OVA $257-264$ (SIINFEKL) in combination with

148 agonistic $\alpha \mathrm{CD} 40$ and polyl:C, mice were able to substantially control tumor growth (figure S1).

149 To ask whether this immunization regimen was effective in established tumors and if tumor

150 control required booster immunizations, we gave a single vaccination of $\alpha C D 40$, polyl:C, and

151 ovalbumin once tumors were established at ten days post implantation. This treatment

152 approach resulted in significantly smaller tumors compared to controls (figure $1 \mathrm{~A}$ ), affirming

153 the efficacy of the vaccination approach. However, a single vaccination at day ten was

154 insufficient to abolish tumor growth, and therefore provided the opportunity to investigate

155 how vaccination alters the antitumor immune response.

156 The antigen component of the vaccine includes epitopes recognized by both CD8 and CD4 T

157 cells, so we measured the number of both intratumoral T cell subsets. Initial studies were

158 performed at eleven days post-vaccination (day 21 after tumor implantation), when we

159 observed reduced tumor size and sufficient time has passed for T cells to be activated and

160 traffic to the tumors. Vaccination increased the number of OVA 257 specific CD8 (12 fold) as well

161 as total antigen experienced CD8 ( 8 fold) and CD4 ( 8 fold) T cells present in the tumors (figure

162 1B). Since vaccination with the OVA protein increased both CD8 and CD4 intratumoral T cells,

163 we used depleting antibodies to test the relative contribution of CD8 versus CD4 T cells to

164 delaying tumor growth. Tumor control after vaccination was lost when both CD8 and CD4 T

165 cells were depleted, but either subset alone was sufficient to delay tumor growth (figure 1C). 
Pre-existing intratumoral CD8 T cells substantially contribute to control tumors following therapeutic anti-CD40 and polyl:C based vaccination

166 Thus, both CD8 and CD4 T cells can contribute to the control of tumor after $\alpha \mathrm{CD} 40$ and polyl:C

167 mediated vaccination with antigen containing epitopes for both subsets.

\section{Additional T cell infiltration is not required for vaccination to slow tumor growth}

As the spontaneous anti-tumor immune response in untreated mice has a limited ability to control B16cOVA outgrowth, we would predict that vaccination slows tumor growth through

171 the recruitment of more functional T cells to tumors. Alternatively, vaccination could lead to

172 expansion and/or improved function in the T cells that have already infiltrated the tumor,

173 despite their apparent exhausted phenotype in this model.(28) To test the contribution of new

174 T cell infiltration to tumor control, we used the sphingosine-1-phosphate receptor agonist

175 FTY720 to block T cell egress from lymphoid tissues and thereby block new T cell trafficking into

176 tumors (figure 2A,S2).(29) Delaying FTY720 treatment allowed the initial antitumor T cell

177 response to infiltrate the tumor prior to vaccination. Contrary to our expectation, vaccination

178 still delayed tumor growth when FTY720 was used to block trafficking of additional T cells into

179 the tumor (figure 2B). Separately, we prevented new T cell trafficking into tumors with $\alpha$ LFA1,

180 which blocks T cell extravasation through LFA1 interaction with ICAM on vasculature.(30)

181 Tumors in mice treated with $\alpha$ LFA1 and vaccinated grew slower than those treated with $\alpha$ LFA1

182 alone (figure S3A). Consistent with reports that $\alpha$ LFA1 alters T cell expansion and function in

183 addition to blocking trafficking,(31,32) we observed decreased expansion of antigen specific T

184 cells in the spleens of vaccinated tumor bearing mice (figure S3B). Despite the diminished T cell

185 expansion, aLFA1 did not prevent vaccination from delaying tumor growth. Together, these 
Pre-existing intratumoral CD8 T cells substantially contribute to control tumors following therapeutic anti-CD40 and polyl:C based vaccination data show that this vaccine can delay tumor growth through T cells that infiltrated the tumor prior to treatment without the need for recruitment of de novo activated T cells.

The vaccines ability to slow tumor growth through the pre-infiltrating $T$ cells suggests that vaccination is acting within the tumor. In support of this, we found that intratumoral DC expressed significantly more CD86 two days after vaccination and only expressed IL12 (p40) in vaccinated mice (figure S4). Thus, intratumoral DC may serve as the intermediary linking vaccination and tumor control dependent on the already infiltrating $T$ cells, and is consistent with previous reports demonstrating that intratumoral DC are critical contributors to

194 vaccination-induced tumor control. $(33,34)$

\section{Vaccination sustains T cells within the tumor}

We next considered how vaccination could slow tumor growth without new T cell infiltration.

The two most likely explanations are that vaccination expanded the T cells within the tumor, or that vaccination improved the function of the T cells within the tumor. The number of OVA 257 specific (9-fold) and total antigen experienced (9-fold) intratumoral CD8 T cells was higher at day eleven after vaccination when trafficking was limited with FTY720 (figure 2C). The number

201 of CD4 T cells was also increased in vaccinated tumors (4-fold), although to a lesser extent than 202 the CD8 T cells (figure 2 C). This indicates that vaccination expanded or preserved both CD8 and

203 CD4 T cells within the tumor. Surprisingly, we found similar numbers of all three groups of

204 intratumoral T cells after vaccination with or without blocking T cell trafficking (figure S5A).

205 This finding, when considered with the subdued expansion of vaccine-specific T cells in the 
Pre-existing intratumoral CD8 T cells substantially contribute to control tumors following therapeutic anti-CD40 and polyl:C based vaccination spleens of tumor-bearing mice (figure S6), indicates that vaccination primarily affects T cells within the tumor rather than driving additional T cell infiltration.

To further understand how vaccination increased the number of both CD8 and CD4 T cells without new infiltration, we measured the number of intratumoral T cells from two to eleven days after vaccination while trafficking was blocked with FTY720. Whereas untreated tumors

211 had fewer $\mathrm{OVA}_{257}$ specific CD8 T cells as time progressed, the number of OVA 257 specific CD8 T

212 cells remained steady in the vaccinated tumors (figure 3A). Although there were fewer CD4 T

213 cells than $\mathrm{OVA}_{257}$ specific CD8 T cells, they followed the same pattern: sustained numbers in

214 vaccinated tumors and a decline over time in unvaccinated tumors (figure 3E). To determine

215 how vaccination sustained intratumoral T cell numbers, we measured proliferation and survival

216 of the T cells. There was an increase in the proportion of OVA specific CD8 expressing Ki67 at

217 four days after vaccination but a decrease in Ki67 expression at seven and eleven days after

218 vaccination, indicating a transient increase in proliferation (figure 3B). Similarly, more CD4 T

219 cells expressed Ki67 at four days, and fewer at eleven days after vaccination (figure 3F). To

220 examine survival, we measured expression of $\mathrm{Bcl} 2$ and caspase $3 / 7$ activation. Both OVA 257

221 specific CD8 and total CD4 T cells had no difference in Bcl2 expression at two and four days

222 after vaccination and a trending decrease in at seven days after vaccination (figure $3 C, G$ ).

223 Similar activated caspase 3/7 levels indicated no difference in cell death in either OVA 257

224 specific CD8 or total CD4 T cells at seven days after vaccination (figure 3D,H). Together, the

$225 \mathrm{Bcl} 2$ and caspase 3/7 data indicate that vaccination does not improve intratumoral T cell

226 survival. In summary, vaccination drives increased proliferation of intratumoral T cells acutely,

227 which may contribute to sustained numbers of both CD8 and CD4 T cells within the tumor. 
Pre-existing intratumoral CD8 T cells substantially contribute to control tumors following therapeutic anti-CD40 and polyl:C based vaccination

228 Vaccination does not improve T cell function within the tumor

229 Aside from T cell infiltration, we also predicted that vaccination would improve the function of

230 intratumoral T cells. Supporting this, $11 \mathrm{~d}$ after vaccination we found that the intensity of

231 expression of the inhibitory receptor PD-1, which can lead to diminished T cell function when

232 engaged by its ligands PD-L1 or PD-L2 on tumor or other immune cells in the tumor

233 microenvironment, was significantly lower ( 2 -fold) on a per cell basis on OVA 257 specific CD8 T

234 cells from vaccinated mice (figure S7A). To test function, we isolated OVA 257 specific CD8 T cells

235 from vaccinated and unvaccinated tumors and co-cultured them in vitro with OVA 257 -pulsed

236 antigen presenting cells. Contrary to our expectations, significantly fewer OVA257 specific CD8 T

237 cells from the tumors of vaccinated mice produced IFN $\gamma$ and Granzyme B than those present in

238 the tumors of control mice (figure S7B,C). Thus, diminished PD-1 expression did not lead to

239 increased effector function of OVA 257 specific CD8 T cells after vaccination. We also compared

240 T cell function after vaccination when trafficking was uninhibited. Remarkably, fewer OVA 257

241 specific CD8 T cells from vaccinated mice produced IFN $\gamma$ than from unvaccinated mice when

242 trafficking was allowed (figure S5B), although Granzyme B expression was not significantly

243 different (figure S5C). This suggests that after vaccination newly infiltrating $\mathrm{T}$ cells are more

244 cytotoxic but not more capable of IFN $y$ production compared to T cells already present within

245 the tumor.

246 An inflection point in tumor growth occurs around ten days after vaccination, which could

247 suggest that the effects of a single vaccination are only temporary. Therefore, we examined

248 the function of both CD8 and CD4 T cells at earlier time points after vaccination. We used 
Pre-existing intratumoral CD8 T cells substantially contribute to control tumors following therapeutic anti-CD40 and polyl:C based vaccination

249 FTY720 to block trafficking of newly primed T cells and harvested tumors at 4 and 7 days after

250 vaccination. In this instance, we gave brefeldin A (BFA) i.v. prior to harvest to block cytokine

251 secretion in situ and thus measure cytokine production by T cells within the tumors. While

252 there was no difference in effector activity in T cells present in the tumors of vaccinated and

253 control mice at day four, a lower proportion of OVA 257 specific CD8 produced IFNY ( 50\%

254 reduction) and Granzyme B ( 30\% reduction) within the tumor seven days after vaccination

255 (figure 4A-C). As in vivo BFA reports steady state cytokine production and may reflect lack of

256 encounter with antigen-bearing cells, we also measured the T cell functional activity by

257 stimulating the T cells isolated from tumors with a low concentration of $\alpha \mathrm{CD} 3$ ex vivo to directly

258 engage the T cell receptors. There was a trend for a higher frequency of OVA $257-S p e c i f i c$ CD8 T

259 cells expressing IFN $\gamma$ at day four but, as seen with in situ assessment, a significant reduction at

260 day seven after vaccination (figure S8A). Ex vivo stimulation also allowed us to assess the

261 capability of the T cells to degranulate, which is required for release of cytotoxic molecules such

262 as Granzyme B. There was no difference in the capability of OVA 257 specific CD8 T cells to

263 degranulate as measured by surface exposure of CD107a during the ex vivo stimulation (figure

264 S8B). This suggests that vaccination did not alter the cytotoxicity of OVA 257 specific CD8 T cells.

265 Additionally, we saw the same pattern of decreased effector function in intratumoral CD8 T

266 cells that were not specific for OVA 257 after vaccination, both in situ and ex vivo (not shown).

267 This demonstrates that vaccination had similar effects on all intratumoral CD8 T cells regardless

268 of the actual antigen they recognized, and further supports that vaccination is primarily

269 affecting the intratumoral T cells. Together, this data shows that vaccination is not controlling

270 tumor growth by improving CD8 T cell effector molecule expression or degranulation. 
Pre-existing intratumoral CD8 T cells substantially contribute to control tumors following therapeutic anti-CD40 and polyl:C based vaccination

271 While CD8 T cells are important based on their cytotoxic capabilities, CD4 T cells can contribute

272 to the antitumor response through cytokines, and in some cases killing of target cells.

273 Therefore, we also examined the function of CD4 T cells within the tumor. In situ, intratumoral

274 CD4 T cells had no significant difference in either IFN $\gamma$ or Granzyme B expression at day four or

275 day seven after vaccination compared to controls (figure 4D,E). Intratumoral CD4 T cells also

276 showed no difference in degranulation based on surface exposure of CD107a during ex vivo

277 stimulation with $\alpha$ CD3 (figure S8D). In contrast to both OVA 257 specific CD8 and CD4 T cell in

278 situ production of IFN $\gamma$, more CD4 T cells expressed IFN $\gamma$ at both four and seven days after

279 vaccination when stimulated ex vivo (figure S8C). Although the vaccinated CD4 T cells did not

280 make more IFN $\gamma$ within the tumor, they have increased potential to contribute effector

281 cytokines.

282 Together these data show that vaccination differentially affects CD8 and CD4 T cell function,

283 and that these effects are discrete when examined in the steady state as compared to after

284 restimulation. Importantly, the relative increase in the number of CD8 and CD4 T cells within

285 the tumor found after vaccination substantially outweighs the reduction in function observed in

286 situ, resulting in equivalent or significantly more effector T cells within the tumors of vaccinated

287 mice (figure S9A-F), especially at d11 post vaccination.

288 Vaccination does not expand T cell subsets identified by Eomes or Tcf1 expression

289 We were intrigued by the reduced function of the vaccinated TIL. Recently, populations of

290 exhausted CD8 T cells that can proliferate and generate more functional CD8 T cells have been

291 separately identified based on two transcription factors: low expression of Eomes and high 
Pre-existing intratumoral CD8 T cells substantially contribute to control tumors following therapeutic anti-CD40 and polyl:C based vaccination expression of Tcf1.(35-37) Therefore, we asked whether a subset of intratumoral CD8 T cells

identified by Eomes or Tcf1 expression was responding to vaccination. We found decreased

Eomes expression in $\mathrm{OVA}_{257}$ specific CD8 T cells from vaccinated tumors at day 17 (figure 5A).

However, unlike published data, we found that T cells with higher Eomes expression had the

305 However, the increase in intratumoral T cells that accompanies vaccination does not result

306 from expansion of a subset of intratumoral CD8 T cells expressing Eomes or Tcf1.

\section{Pre-infiltrated CD8 T cells are sufficient for vaccination to delay tumor growth}

308 Although vaccination sustained both CD8 and CD4 T cell numbers within the tumors, there were

309 more OVA257 specific and total CD8 T cells per tumor (figure 3A,E). However, CD8 had

311 (figure 4,S8). Further, when T cell trafficking was unrestricted, either CD8 or CD4 T cells were

312 sufficient to slow tumor growth (figure 1C). Since additional T cell infiltration was not required 
Pre-existing intratumoral CD8 T cells substantially contribute to control tumors following therapeutic anti-CD40 and polyl:C based vaccination

313 for vaccination to delay tumor growth (figure $2 \mathrm{~A}$ ), we next assessed if both the pre-infiltrated

314 CD8 and CD4 T cells had the potential to impede tumor growth. To test this, we combined T

315 cell depletion with FTY720 to block trafficking into the tumor (figure 6A). As expected, based

316 on our earlier depletion experiment, depleting both CD8 and CD4 T cells in FTY720 treated mice

317 resulted in the loss of vaccination induced tumor control (figure 6B). When we depleted only

318 CD4 cells in FTY720 treated mice, vaccination still delayed tumor growth. However, when we

319 blocked trafficking with FTY720 and depleted CD8 T cells, the already infiltrating CD4 T cells

320 were insufficient to slow tumor growth after vaccination. Thus, when T cell trafficking to tumor

321 is prevented, only the pre-infiltrated CD8 T cells could slow tumor growth. Although the

322 intratumoral CD8 T cells exhibited lower levels of effector functions after vaccination, they still

323 had an essential role. Thus, the CD8 T cells infiltrating the tumor at the time of vaccination play

324 critical contributions to the ability of vaccination to delay tumor growth.

325 DISCUSSION

326 Previously, and in our data, vaccination regimens with $\alpha \mathrm{CD} 40$, polyl:C, and antigen control

327 tumor growth and generate large T cell responses observed in the spleen and blood. As T cells

328 are known to become exhausted within the tumor, it was assumed that newly activated T cells

329 were trafficking to the tumor and responsible for controlling tumor growth. However, the

330 relative increase in the numbers of T cells in tumors after vaccination was greater than seen in

331 the periphery, and this relative increase was sustained when trafficking from the periphery was

332 blocked. Further, by blocking trafficking we have shown that the existing CD8 T cells are

333 sufficient for vaccination to slow tumor growth without additional T cell infiltration. Together, 
Pre-existing intratumoral CD8 T cells substantially contribute to control tumors following therapeutic anti-CD40 and polyl:C based vaccination

334 these data make the unexpected argument that pre-existing intratumoral T cells are critical

335 responders to this vaccine regimen.

336 Despite the compelling evidence that vaccination leads to tumor control via pre-existing

337 intratumoral T cells, there are several paradoxes concerning the mechanistic basis of tumor

338 control. First, we predicted that vaccination would lead to more functional T cells within the

339 tumor. Instead, a lower proportion of CD8 T cells from vaccinated tumors expressed IFN $\gamma$ and

340 Granzyme B in situ acutely after vaccination, and they were no better at degranulating. While a

341 greater proportion of CD4 T cells from vaccinated tumors had the potential to produce more

342 IFNy ex vivo, they were also not more functional in situ within the tumor. Intriguingly, ex vivo

343 activity more closely correlated with tumor control, suggesting that relatively few tumor-

344 specific T cells are encountering antigen at any given time within tumors or that they are being

345 actively suppressed. The basis for the loss of effector activity by the CD8 tumor infiltrating

346 lymphocytes 7-11d after vaccination remains unclear. OVA 257 specific CD8 T cells expressed less

347 PD-1, which we would expect to improve function within the tumor. Decreased Eomes

348 expression may have contributed to diminished function. However, T-bet, which was highly

349 expressed (not shown), and Tcf1 levels were unaffected by vaccination. Glycolytic activity in T

350 cells has been shown to regulate IFN $\gamma$ translation,(38) and we have previously shown that

351 tumor infiltrating lymphoyctes have limited glycolysis.(28) We are currently investigating

352 whether vaccination leads to a further reduction in glycolytic activity in intratumoral T cells.

353 Notably, intratumoral T cells from vaccinated mice with intact trafficking were also less

354 functional than from control mice, suggesting that loss of functional activity occurs within the

355 tumor microenvironment. Critically, however, the decreased function of intratumoral T cells 
Pre-existing intratumoral CD8 T cells substantially contribute to control tumors following therapeutic anti-CD40 and polyl:C based vaccination was offset by vaccination sustaining $T$ cell numbers within the tumors, resulting in an equivalency or increase in the number of cytokine expressing T cells per tumor, even when gauged by in situ function (figure $S 9 A, B, D, E)$. Thus, the impact of vaccination on $T$ cell persistence in the tumor mitigates their diminished functionality. It is possible that other effector mechanisms or aspects of T cell biology that we currently do not fully understand are contributing to tumor control. and CD4 T cells present in the tumor even when trafficking of T cells into the tumor is blocked.

364 This suggests, surprisingly, that the sustained intratumoral T cell numbers are a function of maintaining or expanding the existing T cells rather than new T cell trafficking to tumors. While there was increased proliferation of intratumoral T cells acutely after vaccination, $\mathrm{T}$ cells within

367 the tumor were less proliferative at later time points. Nevertheless, there could be a subset of

368 T cells proliferating in response to vaccination that is missed by considering the total

369 population. To address this possibility, we focused on two transcription factors known to be 370 critical for T cell proliferation in a tumor setting, Eomes and Tcf1. Expression of both Eomes 371 and Tcf1 correlated with increased proliferation, yet vaccination did not affect Tcf1 and led to 372 reduced Eomes expression in intratumoral CD8 T cells, and did not improve proliferation in a 373 Tcf1+ or Eomes+ subset. T cells from vaccinated mice expressed less of the survival protein $374 \mathrm{Bcl} 2$, but had equivalent caspase $3 / 7$ activation. Together, these data suggest neither 375 expansion of less differentiated T cells nor increased survival were responsible for sustaining T 376 cell numbers in response to vaccination. We also considered retention of T cells in the tumor.

377 However, we found that the majority of OVA specific T cells expressed the retention integrin 
Pre-existing intratumoral CD8 T cells substantially contribute to control tumors following therapeutic anti-CD40 and polyl:C based vaccination

378 CD103 with no difference after vaccination (not shown). Thus, we currently ascribe the impact

379 of vaccination on T cell numbers to the acute burst in proliferation, but why proliferation is

380 rapidly curtailed remains to be determined.

381 These mechanistic paradoxes are important as $\alpha$ CD40 and TLR3 agonists are being aggressively

382 developed for clinical implementation and suggest that efficacy may be divorced from

383 traditional biomarkers of tumor control. A combination of agonistic $\alpha C D 40$, the polyl:C

384 derivative polyIC:LC, and peptides generated a substantial antigen specific CD8 response in

385 nonhuman primates.(39) CD40 monoclonal antibodies have shown to be safe in human trials,

386 with limited efficacy on tumor control as a single agent,(40-42) and better response rates

387 when combined with chemotherapy or checkpoint blockade.(43,44) PolyIC:LC has been

388 incorporated into human vaccines that generate T cell responses but not necessarily control

389 tumor growth $(8,9)$. Two clinical trials with $\alpha$ CD40, polyIC:LC, and peptide antigens have been

390 initiated for melanoma patients (NCT03597282, NCT04364230), but have not reported

391 outcomes. Based on our data, the existing T cell response may be critical for such vaccination

392 regimens to be effective. Additionally, our data suggests that circulating T cells are not a

393 definitive biomarker for vaccination efficacy, as they are not required for tumor control.

394 In our model, vaccination was administered at a site distant to the tumor, yet CD8 T cells that

395 were already present within the tumor were sufficient for tumor control. This suggests that

396 vaccine administration directly into the tumor could have the same effects within the tumor,

397 and likely at much lower doses, which could limit systemic toxicity. Additionally, since

398 vaccination affected T cells within the tumor, the antigen component may not be necessary. 
Pre-existing intratumoral CD8 T cells substantially contribute to control tumors following therapeutic anti-CD40 and polyl:C based vaccination

However, when we treated mice with $\alpha \mathrm{CD} 40$ and polyl:C without additional antigen plus

FTY720 to block trafficking, only half the mice were able to delay tumor growth (not shown).

Mice that responded had equivalent tumor control as mice that received the full vaccination,

suggesting that the amount of tumor-derived antigen presented by intratumoral DC is

insufficient in non-responder mice. This argues for including either shared antigens or

neoantigens identified by sequencing tumors in human vaccination regimens. Alternatively,

405 treatments that liberate tumor antigens could be combined with $\alpha$ CD40 and polyIC:LC, and this

406 may account for the cases where $\alpha \mathrm{CD} 40$ and chemotherapy were successfully combined in

407 patients. Future studies in these areas would provide additional clarity and potential

408 effectiveness for translating vaccination with $\alpha \mathrm{CD} 40$ and polyl:C to human cancer patients.

\section{CONCLUSIONS}

410 Vaccination with $\alpha \mathrm{CD} 40$, polyl:C, and tumor antigen can delay the growth of established

411 melanoma tumors by proficiently sustaining pre-infiltrating CD8 T cells. Intratumoral T cells

412 lose effector function with time after vaccination, yet are more acutely proliferative resulting in

413 a relative increase in the number of effector T cells within the tumors of vaccinated mice.

414 Although vaccination drives T cell expansion in the periphery, our data shows that additional T

415 cell infiltration is unnecessary for tumor control, and that the existing intratumoral CD8 T cells

416 therefore critically contribute to tumor control after this vaccination regime.

\section{ACKNOWLEDGEMENTS}


Pre-existing intratumoral CD8 T cells substantially contribute to control tumors following therapeutic anti-CD40 and polyl:C based vaccination

418 The authors would like to thank Dr. Alexandra Witter and Ms. Marissa Gonzales for assistance

419 and discussions and the Beirne B. Carter Center for Immunology Research for use of the flow

420 cytometry instruments.

\section{REFERENCES}

422 1. Erdag G, Schaefer JT, Smolkin ME, Deacon DH, Shea SM, Dengel LT, et al. Immunotype and immunohistologic characteristics of tumor-infiltrating immune cells are associated with clinical outcome in metastatic melanoma. Cancer Res. 2012 Mar 1;72(5):1070-80.

2. Tumeh PC, Harview CL, Yearley JH, Shintaku IP, Taylor EJM, Robert L, et al. PD-1 blockade induces responses by inhibiting adaptive immune resistance. Nature. 2014 Nov

3. Jäeger E, Bernhard H, Romero P, Ringhoffer M, Arand M, Karbach J, et al. Generation of cytotoxic T-cell responses with synthetic melanoma-associated peptides in vivo: Implications for tumor vaccines with melanoma-associated antigens. Int J Cancer. 1996 Apr 10;66(2):162-9.

4. Cormier JN, Salgaller ML, Prevette T, Barracchini KC, Rivoltini L, Restifo NP, et al. MART-1/Melan A. Cancer J Sci Am. 1997;3(1):37-44. 
Pre-existing intratumoral CD8 T cells substantially contribute to control tumors following therapeutic anti-CD40 and polyl:C based vaccination

438 6. Slingluff CL, Yamshchikov G, Neese P, Galavotti H, Eastham S, Kittlesen D, et al. Phase I trial of a melanoma vaccine with gp100280-288 peptide and tetanus helper peptide in adjuvant: Immunologic and clinical outcomes. Clin Cancer Res. 2001 Oct 1;7(10):301224.

7. Marchand M, Baren N van, Weynants $P$, Brichard V, Dréno B, Tessier $M$, et al. Tumor regressions observed in patients with metastatic melanoma treated with an antigenic peptide encoded by gene MAGE-3 and presented by HLA-A1. Int J Cancer. 1999 Jan

8. Melssen MM, Petroni GR, Chianese-Bullock KA, Wages NA, Grosh WW, Varhegyi N, et al. A multipeptide vaccine plus toll-like receptor agonists LPS or polyICLC in combination with incomplete Freund's adjuvant in melanoma patients. J Immunother Cancer. 2019

9. Pavlick A, Blazquez AB, Meseck M, Lattanzi M, Ott PA, Marron TU, et al. Combined vaccination with NY-ESO-1 protein, poly-ICLC, and montanide improves humoral and cellular immune responses in patients with high-risk melanoma. Cancer Immunol Res. 2020 Nov 7;8(1):70-80.

10. Callan MFC, Tan L, Annels N, Ogg GS, Wilson JDK, O'Callaghan CA, et al. Direct visualization of antigen-specific CD8+ T cells during the primary immune response to Epstein-Barr virus in vivo. J Exp Med. 1998 May 4;187(9):1395-402. 
Pre-existing intratumoral CD8 T cells substantially contribute to control tumors following therapeutic anti-CD40 and polyl:C based vaccination therapeutic antitumor effects. Cancer Res. 2009 Dec 1;69(23):9012-9.

12. Schoenberger SP, Toes REM, Van Dervoort EIH, Offringa R, Melief CJM. T-cell help for cytotoxic T lymphocytes is mediated by CD40-CD4OL interactions. Nature. 1998 Jun

13. Zaks K, Jordan M, Guth A, Sellins K, Kedl R, Izzo A, et al. Efficient Immunization and CrossLiposomes. J Immunol. 2006;176(12):7335-45.

14. Salem ML, Kadima AN, Cole DJ, Gillanders WE. Defining the Antigen-Specific T-Cell

May;28(3):220-8.

15. Ahonen CL, Doxsee CL, McGurran SM, Riter TR, Wade WF, Barth RJ, et al. Combined TLR and CD40 Triggering Induces Potent CD8+ T Cell Expansion with Variable Dependence on Type I IFN. J Exp Med. 2004 Mar 15;199(6):775-84.

16. Sanchez PJ, McWilliams JA, Haluszczak C, Yagita H, Kedl RM. Combined TLR/CD40 CD70 In Vivo. J Immunol. 2007 Feb 1;178(3):1564-72. CD4 + T Cells . J Immunol. 2005 Jan 15;174(2):710-7. 
Pre-existing intratumoral CD8 T cells substantially contribute to control tumors following therapeutic anti-CD40 and polyl:C based vaccination immunization with adjuvant molecules poly(I:C) and anti-CD40 plus a tumor antigen has potent prophylactic and therapeutic antitumor effects. Cancer Immunol Immunother. 2008 Jan 13;57(1):19-29.

19. Hailemichael Y, Dai Z, Jaffarzad N, Ye Y, Medina MA, Huang XF, et al. Persistent antigen at vaccination sites induces tumor-specific CD8+ T cell sequestration, dysfunction and deletion. Nat Med. 2013 Apr;19(4):465-72.

20. Broomfield SA, van der Most RG, Prosser AC, Mahendran S, Tovey MG, Smyth MJ, et al. Locally Administered TLR7 Agonists Drive Systemic Antitumor Immune Responses That Are Enhanced by Anti-CD40 Immunotherapy. J Immunol. 2009 May 1;182(9):5217-24.

21. Bialojan A, Sohl J, Rausch J, Aranda Lopez P, Denny M, Langguth P, et al. Transcutaneous immunization with CD40 ligation boosts cytotoxic T lymphocyte mediated antitumor immunity independent of CD4 helper cells in mice. Eur J Immunol. 2019;49(11):2083-94.

22. Lum HD. In vivo CD40 ligation can induce T cell-independent antitumor effects that involve macrophages. J Leukoc Biol. 2006 Mar 30;79(6):1181-92.

23. Medina-Echeverz J, Ma C, Duffy AG, Eggert T, Hawk N, Kleiner DE, et al. Systemic agonistic anti-CD40 treatment of tumor-bearing mice modulates hepatic myeloidsuppressive cells and causes immune-mediated liver damage. Cancer Immunol Res. 2015 May 1;3(5):557-66. agonists alter tumor stroma and show efficacy against pancreatic carcinoma in mice and 
Pre-existing intratumoral CD8 T cells substantially contribute to control tumors following therapeutic anti-CD40 and polyl:C based vaccination humans. Science (80- ). 2011 Mar 25;331(6024):1612-6.

25. Barrios K, Celis E. TriVax-HPV: An improved peptide-based therapeutic vaccination strategy against human papillomavirus-induced cancers. Vol. 61, Cancer Immunology, Immunotherapy. Springer; 2012. p. 1307-17.

26. Hwang ML, Lukens JR, Bullock TNJ. Cognate Memory CD4 + T Cells Generated with Dendritic Cell Priming Influence the Expansion, Trafficking, and Differentiation of Secondary CD8 + T Cells and Enhance Tumor Control . J Immunol. 2007 Nov 1;179(9):5829-38.

27. Roberts DJ, Franklin NA, Kingeter LM, Yagita H, Tutt AL, Glennie MJ, et al. Control of Established Melanoma by CD27 Stimulation Is Associated With Enhanced Effector Function and Persistence, and Reduced PD-1 Expression of Tumor Infiltrating CD8+ T Cells. J Immunother. 2010 Oct;33(8):769-79.

28. Gemta LF, Siska PJ, Nelson ME, Gao X, Liu X, Locasale JW, et al. Impaired enolase 1 glycolytic activity restrains effector functions of tumor-infiltrating CD8+ T cells. Sci Immunol. 2019 Jan 25;4(31).

29. Brinkmann V, Davis MD, Heise CE, Albert R, Cottens S, Hof R, et al. The immune modulator FTY720 targets sphingosine 1-phosphate receptors. J Biol Chem. 2002 Jun $14 ; 277(24): 21453-7$.

30. Woods AN, Wilson AL, Srivinisan N, Zeng J, Dutta AB, Peske JD, et al. Differential expression of homing receptor ligands on tumor-associated vasculature that control CD8 
Pre-existing intratumoral CD8 T cells substantially contribute to control tumors following therapeutic anti-CD40 and polyl:C based vaccination effector T-cell entry. Cancer Immunol Res. 2017 Dec 1;5(12):1062-73.

31. Rutigliano JA, Johnson TR, Hollinger TN, Fischer JE, Aung S, Graham BS. Treatment with Anti-LFA-1 Delays the CD8+ Cytotoxic-T-Lymphocyte Response and Viral Clearance in Mice with Primary Respiratory Syncytial Virus Infection. J Virol. 2004 Mar 15;78(6):3014-

23.

32. Setoguchi K, Schenk AD, Ishii D, Hattori Y, Baldwin WM, Tanabe K, et al. LFA-1 antagonism inhibits early infiltration of endogenous memory CD8 T cells into cardiac allografts and donor-reactive T cell priming. Am J Transplant. 2011 May;11(5):923-35.

33. Pulko V, Liu X, Krco CJ, Harris KJ, Frigola X, Kwon ED, et al. TLR3-Stimulated Dendritic Cells Up-regulate B7-H1 Expression and Influence the Magnitude of CD8 T Cell Responses to Tumor Vaccination. J Immunol. 2009 Sep 15;183(6):3634-41.

34. Azuma M, Takeda Y, Nakajima H, Sugiyama H, Ebihara T, Oshiumi H, et al. Biphasic function of TLR3 adjuvant on tumor and spleen dendritic cells promotes tumor T cell infiltration and regression in a vaccine therapy. Oncoimmunology. 2016 Aug

35. Paley MA, Kroy DC, Odorizzi PM, Johnnidis JB, Dolfi D V., Barnett BE, et al. Progenitor and terminal subsets of CD8+ T cells cooperate to contain chronic viral infection. Science (802;5(8):e1188244. that provide the proliferative burst after PD-1 therapy. Nature. 2016 Aug 
Pre-existing intratumoral CD8 T cells substantially contribute to control tumors following therapeutic anti-CD40 and polyl:C based vaccination 2;537(7620):417-21.

37. Siddiqui I, Schaeuble K, Chennupati V, Fuertes Marraco SA, Calderon-Copete S, Pais

Ferreira D, et al. Intratumoral Tcf1 + PD-1 + CD8 + T Cells with Stem-like Properties

Promote Tumor Control in Response to Vaccination and Checkpoint Blockade

Immunotherapy. Immunity. 2019 Jan 15;50(1):195-211.e10.

38. Chang CH, Curtis JD, Maggi LB, Faubert B, Villarino A V., O'Sullivan D, et al.

Posttranscriptional control of T cell effector function by aerobic glycolysis. Cell. 2013 Jun

6;153(6):1239.

39. Thompson EA, Liang F, Lindgren G, Sandgren KJ, Quinn KM, Darrah PA, et al. Human Antithe Lung of Nonhuman Primates. J Immunol. 2015 Aug 1;195(3):1015-24.

40. Vonderheide RH, Flaherty KT, Khalil M, Stumacher MS, Bajor DL, Hutnick NA, et al. novel CD40 agonist monoclonal antibody. J Clin Oncol. 2007 Mar 1;25(7):876-83.

41. Sanborn RE, Gabrail NY, Bhardwaj N, Gordon MS, O'Hara M, Khalil D, et al. Abstract LB194: First-in-human Phase I study of the CD40 agonist mAb CDX-1140 and in combination with CDX-301 (rhFLT3L) in patients with advanced cancers: Interim results. In: Cancer Research. American Association for Cancer Research (AACR); 2019. p. LB-194-LB-194. 
Pre-existing intratumoral CD8 T cells substantially contribute to control tumors following therapeutic anti-CD40 and polyl:C based vaccination tumors. J Clin Oncol. 2018 May 20;36(15_suppl):3093-3093.

43. Vonderheide RH, Burg JM, Mick R, Trosko JA, Li D, Shaik MN, et al. Phase i study of the CD40 agonist antibody CP-870,893 combined with carboplatin and paclitaxel in patients with advanced solid tumors. Oncoimmunology. 2013 Jan;2(1).

44. Bajor DL, Mick R, Riese MJ, Huang AC, Sullivan B, Richman LP, et al. Long-term outcomes of a phase I study of agonist CD40 antibody and CTLA-4 blockade in patients with metastatic melanoma. Oncoimmunology. 2018 Oct 3;7(10).

\section{FIGURE LEGENDS}

Figure 1. Therapeutic efficacy of vaccination with $\alpha \mathrm{CD} 40 /$ polyl:C/antigen in murine melanoma. on day 10. $n=6$ mice per group. (B) Relative number of $\mathrm{OVA}_{257}$ specific CD8 T cells identified by MHC I dextramer (top) and total antigen experienced CD8 (middle) and CD4 (bottom) T cells

570 infiltrating tumors at day 21 from mice vaccinated as in (A). Data are pooled from five

571 experiments and normalized to the number of T cells in unvaccinated control tumors within

572 each experiment. (C) Tumor size in mice vaccinated at day 10 as in (A) and injected i.p. with 573 depleting antibodies to CD8 and/or CD4 every four days starting at day 8. n=4-7 mice per group.

574 Data were analyzed by Holm-Sidak multiple t tests (AC) or Welch's t test (B). (C) Significant 575 difference between Control/Vax, Control/Vax $+\alpha C D 8$, and Control/Vax $+\alpha C D 4$ at indicated time points. ${ }^{*} p<0.05, * * p<0.01$ 
Pre-existing intratumoral CD8 T cells substantially contribute to control tumors following therapeutic anti-CD40 and polyl:C based vaccination

577 Figure 2. Additional T cell infiltration is not required for vaccination to slow tumor growth. (A)

578 Mice bearing B16cOVA tumors were vaccinated and treated with FTY720 as indicated in the

579 timeline and described in the methods. (B) Tumor size of mice treated with vaccination and

580 FTY720. $n=5$ per group. (C) Relative number of OVA 257 specific CD8 T cells (top) and total

581 antigen experienced CD8 (middle) and CD4 (bottom) T cells infiltrating tumors at day 21. Data

582 are pooled from 2 experiments. Each data point is from an individual mouse. Data were

583 analyzed by Holm-Sidak multiple t tests (B) or Welch's t test (C). (B) Significant difference

584 between Control/Vax, Control/Vax+FTY, FTY/Vax, and FTY/FTY+Vax at indicated time points.

$585 * p<0.05, * * p<0.01$

Figure 3. Vaccination sustains T cells within the tumor. Mice bearing B16cOVA tumors were vaccinated at day 10 and began receiving FTY720 at day 9 as described in methods and shown

588 in figure 2A. Tumors were harvested and T cells were analyzed at 2, 4, 7, or 11 days after

589 vaccination or from control mice at matching time points. (AE) Number of intratumoral OVA 257

590 specific CD8 (A) and CD4 (E) T cells. (BF) Relative proportion of Ki67 expressing OVA 257 specific

591 CD8 (B) and CD4 (F) T cells within control or vaccinated tumors. (CG) Relative per cell expression

592 of Bcl2 in intratumoral OVA 257 specific CD8 (C) and CD4 (G) T cells. (DH) Relative caspase 3/7

593 activation in intratumoral OVA 257 specific CD8 (D) and CD4 (H) T cells. Data were analyzed by

594 ANOVA with test for linear trend (AE) or Holm-Sidak multiple t tests(B-D,F-H). ${ }^{*} p<0.05$,

$595 * * p<0.01, * * * p<0.001, * * * * p<0.0001$

596 Figure 4. Vaccination does not improve T cell function within the tumor. Mice bearing B16cOVA

597 tumors were vaccinated at day 10 and began receiving FTY720 at day 9 as described in methods 
Pre-existing intratumoral CD8 T cells substantially contribute to control tumors following therapeutic anti-CD40 and polyl:C based vaccination and shown in figure $2 \mathrm{~A}$. Brefeldin A was administered 5 hours prior to harvest to measure in vivo expression of IFNY and Granzyme B by tumor infiltrating $\mathrm{OVA}_{257}$ specific CD8 and CD4 T cells. (A) Representative flow plots of naïve T cells from the spleen (top) and OVA 257 specific CD8 or CD4 T cells from control (middle) and vaccinated (bottom) tumors. (BC) Relative proportion of $\mathrm{OVA}_{257}$ specific CD8 T cells expressing IFNY (B) and Granzyme B (C) from 3 pooled experiments. (DE) Relative proportion of CD4 T cells expressing IFN $(D)$ and Granzyme B (E)

604 from 3 pooled experiments. Data were analyzed by Holm-Sidak multiple t tests. ${ }^{*} p<0.05$, 605 $* * p<0.01, * * * p<0.001$

Figure 5. Vaccination does not improve proliferation or function in a CD8 T cell subset identified by Eomes or Tcf1. Mice bearing B16cOVA tumors were vaccinated at day 10 and began receiving FTY720 at day 9 as described in methods and shown in figure 2A. Brefeldin A was

609 administered 5 hours prior to harvest. (A) Expression of Eomes in intratumoral OVA 257 specific

610 CD8 T cells. (B) Expression of Ki67 in in intratumoral OVA 257 specific CD8 T cells that do (blue) or 611 do not (green) express Eomes. (CD) Proportion expressing IFNy (C) and Granzyme B (D) of 612 intratumoral OVA 257 specific CD8 T cells that do (blue) or do not (green) express Eomes. (E) 613 Expression of Tcf1 in intratumoral $\mathrm{OVA}_{257}$ specific CD8 T cells. (F) Expression of Ki67 in in 614 intratumoral OVA 257 specific CD8 T cells that do (orange) or do not (green) express Tcf1. (GH) 615 Proportion expressing IFNY (G) and Granzyme B $(\mathrm{H})$ of intratumoral OVA 257 specific CD8 T cells 616 that do (orange) or do not (green) express Tcf1. Data were analyzed by Holm-Sidak multiple t tests. ${ }^{*} p<0.05, * * p<0.01, * * * p<0.001, * * * * p<0.0001$ 
Pre-existing intratumoral CD8 T cells substantially contribute to control tumors following therapeutic anti-CD40 and polyl:C based vaccination

618 Figure 6. Pre-infiltrated CD8 T cells are sufficient for vaccination to delay tumor growth. (A)

619 Mice bearing B16cOVA tumors were treated with vaccination, FTY720, and depleting antibodies

620 as indicated in the timeline and described in the methods. (B) Tumor size of mice treated as

621 described in (A). n=6-7 per group. Data were analyzed by Holm-Sidak multiple t tests with

622 significant difference between FTY/FTY+Vax and FTY/FTY+Vax+ $\alpha$ CD4 at indicated time points.

$623 * p<0.05$

624 Figure S1. Therapeutic efficacy of double vaccination with $\alpha \mathrm{CD} 40 / \mathrm{poly}(\mathrm{I}: \mathrm{C}) /$ antigen in murine

625 melanoma. (A) Tumor size in mice bearing B16cOVA tumors vaccinated i.p. with

$626 \alpha C D 40 / p o l y l: C / o v a l b u m i n$ on day 3 and 10 or no vaccination control. $n=4-5$ mice per group. (A)

627 Tumor size in mice bearing B16cOVA tumors vaccinated i.p. with $\alpha C D 40 /$ polyl:C/OVA $257-264$ on

628 day 3 and 10 or no vaccination control. $n=3-4$ mice per group. (AB) Data were analyzed by

629 Holm-Sidak multiple t tests. ${ }^{*} p<0.05$.

630

Figure S2. FTY720 blocks T cell trafficking through blood. B16cOVA tumor bearing mice were

631 treated with FTY starting at day 9 and/or vaccinated with $\alpha$ CD40/polyl:C/ovalbumin on day 10.

632 Number of CD8 (A) and CD4 (B) T cells per 100 ul of blood at day 13. Data were analyzed by

633 Welch's t test. ${ }^{*} p<0.05$

634 Figure S3. Effects of $\alpha$ LFA1 on tumor growth and CD8 T cell expansion. (A) Tumor size in mice

635 bearing B16cOVA tumors vaccinated i.p. with $\alpha \mathrm{CD} 40 /$ polyl:C/ovalbumin on day 10 and/or

637 cells were transferred into mice bearing B16cOVA tumors on day 9. Mice were treated with

$638 \alpha \mathrm{LFA} 1$ on day 9, 11, 13, and 15 and/or immunized with $\alpha \mathrm{CD} 40 /$ polyl:C/ovalbumin on day 10. 
Pre-existing intratumoral CD8 T cells substantially contribute to control tumors following therapeutic anti-CD40 and polyl:C based vaccination

639 Spleens were harvested and OT1 T cells were counted at day 17. Data were analyzed by Holm-

640 Sidak multiple t tests (A) or Welch's t test (B). (A) Significant difference between Control and

641 Vax+LFA at indicated time points. ${ }^{*} p<0.05$

642 Figure S4. Vaccination leads to acute intratumoral DC maturation. B16cOVA tumor bearing

643 mice were treated with FTY starting at day 9 and/or vaccinated with aCD40/polyl:C/ovalbumin

644 on day 10. Brefeldin A was administered 5 hours prior to harvest to measure cytokine

645 expression within the tumor. Intratumoral DC were identified as $\mathrm{CD} 11 \mathrm{c}+\mathrm{MHCI}{ }^{\text {hi }}$ cells. (AB)

646 Expression of CD86 by proportion (A) and per cell expression (B) on intratumoral DC. (C) In vivo

647 expression of IL12 by intratumoral DC. (A-C) Data are representative of two experiments. Data

648 were analyzed by Welch's t test. ${ }^{*} p<0.05,{ }^{* *} p<0.01$.

649 Figure S5. Number and function of intratumoral T cells with and without blocked trafficking.

650 B16cOVA tumor bearing mice were treated with FTY starting at day 9 and/or vaccinated with

$651 \alpha C D 40 /$ polyl:C/ovalbumin on day 10. (A) Relative number of OVA 257 specific CD8 T cells (left)

652 and total antigen experienced CD8 (middle) and CD4 (right) T cells infiltrating tumors at day 21.

653 (BC) Expression of IFNY (B) and Granzyme B (C) by tumor infiltrating OVA 257 specific CD8 T cells

654 after in vitro stimulation with $\mathrm{OVA}_{257}$ pulsed splenocytes. (A-B) Data are pooled from two

655 experiments. (A-C) Data were analyzed by Welch's t test. $* p<0.05$.

656 Figure S6. Vaccination expands antigen specific T cells in naïve and tumor bearing mice. Mice

657 were vaccinated with $\alpha \mathrm{CD} 40 /$ polyl:C/ovalbumin in naïve mice or ten days after B16cOVA tumor

658 implantation. Number of OVA 257 specific CD8 T cells present in the spleen seven days after

659 vaccination. Non-tumor and tumor bearing mice were vaccinated in separate experiments. All 
Pre-existing intratumoral CD8 T cells substantially contribute to control tumors following therapeutic anti-CD40 and polyl:C based vaccination

660 groups were significantly different from each other based on Welch's t test with Dunnet's

661 correction.

662 Figure S7. Function of $\mathrm{OVA}_{257}$ specific CD8 T cells at day 11 after vaccination. Mice bearing

663 B16cOVA tumors were vaccinated at day 10 and began receiving FTY720 at day 9 as described

664 in methods and shown in figure 2A. (A) Geometric mean fluorescence of PD1 on tumor

665 infiltrating OVA 257 specific CD8 T cells. (BC) Proportion of tumor infiltrating OVA 257 specific CD8

666 T cells expressing IFN $\gamma(B)$ and Granzyme B (C) after in vitro stimulation with OVA 257 pulsed

667 splenocytes. (A-C) Data are pooled from 2 experiments. Each data point is from an individual

mouse. Data were analyzed by Welch's t test. ${ }^{*} p<0.05,{ }^{*} p<0.01$

669

Figure S8. Ex vivo function of intratumoral T cells. Mice bearing B16cOVA tumors were

670 vaccinated at day 10 and began receiving FTY720 at day 9 as described in methods.

671 Intratumoral T cells were isolated and stimulated with $\alpha \mathrm{CD} 3$ for four hours. (AB) Relative

672 proportion of $\mathrm{OVA}_{257}$ specific CD8 T cells expressing IFNY (A) and CD107a (B). (CD) Relative

673 proportion of CD4 T cells expressing IFNY (C) and CD107a (D). (A-D) Data are pooled from three

674 experiments and normalized to the proportion of positive cells in unvaccinated control tumors

675 within each experiment. Data were analyzed by Holm-Sidak multiple t tests. ${ }^{*} p<0.05,{ }^{*} p<0.01$.

676 Figure S9. Number of functional intratumoral T cells. Mice bearing B16cOVA tumors were

677 vaccinated at day 10 and began receiving FTY720 at day 9 as described in methods and shown

678 in figure 2A. Brefeldin A was administered 5 hours prior to harvest to measure in vivo

679 expression of IFN $y$ and Granzyme B by tumor infiltrating OVA 257 specific CD8 T cells (ABDE) or

680 intratumoral T cells were isolated and stimulated in vitro for four hours (CF). (AB) Relative 
Pre-existing intratumoral CD8 T cells substantially contribute to control tumors following therapeutic anti-CD40 and polyl:C based vaccination

681 number of OVA 257 specific CD8 T cells expressing IFNY (A) and Granzyme B (B) in vivo. (C)

682 Relative number of OVA 257 specific CD8 T cells expressing IFN $\gamma$ ex vivo. (DE) Relative number of

683 CD4 T cells expressing IFN (D) and Granzyme B (E) in vivo. (F) Relative number of CD4T cells

684 expressing IFNy ex vivo. (A-F) Data were analyzed by Holm-Sidak multiple t tests. ${ }^{*} p<0.05$,

685

$* * p<0.01, * * * p<0.001, * * * * p<0.0001$ 
Fig 1

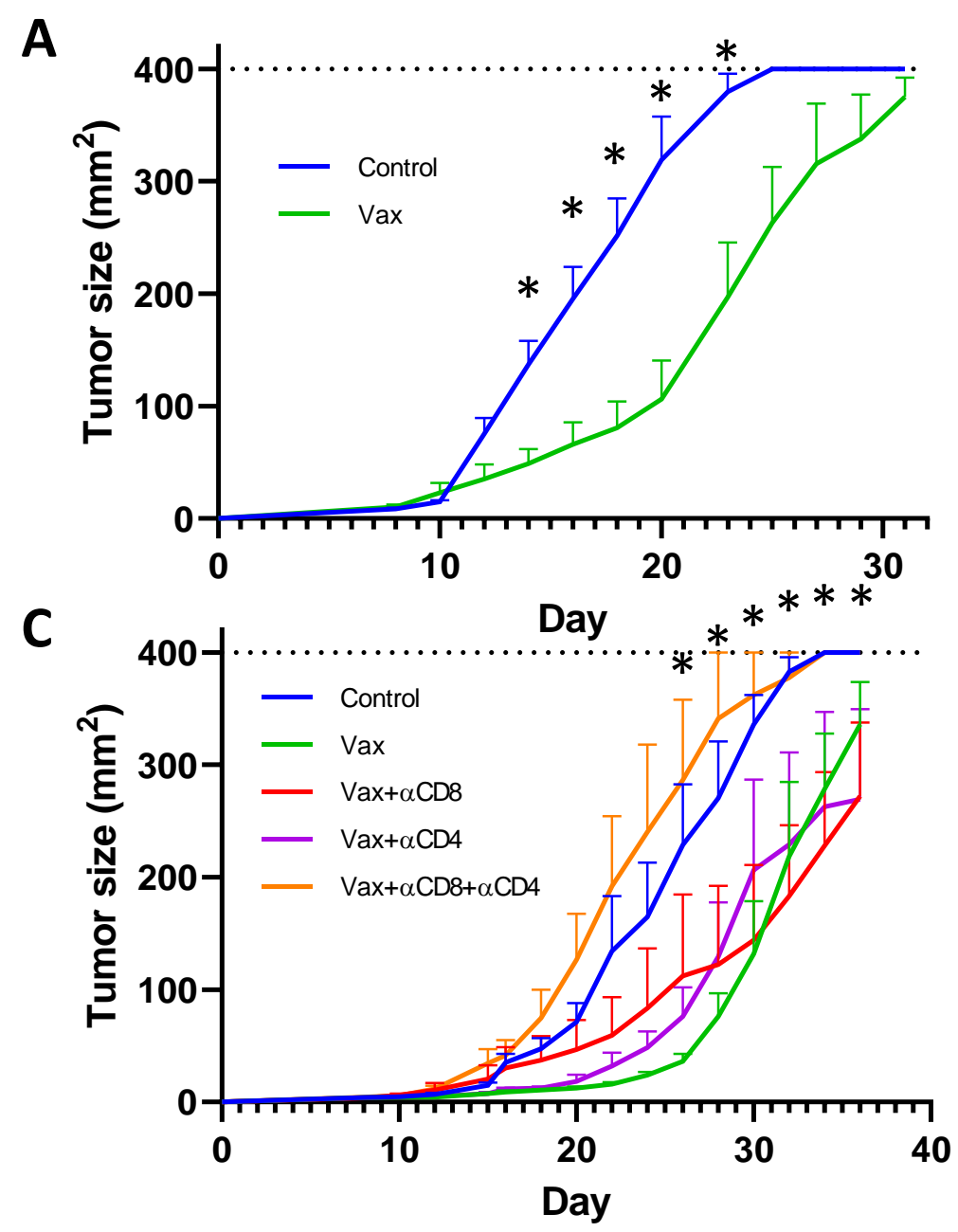

B
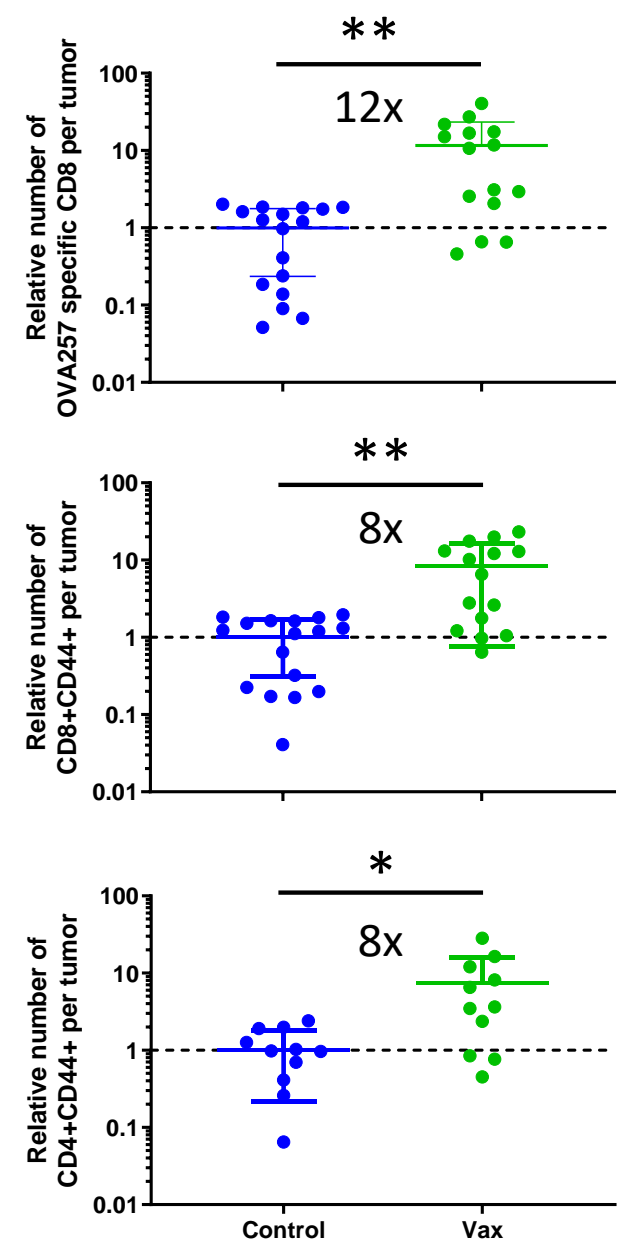
Fig 2
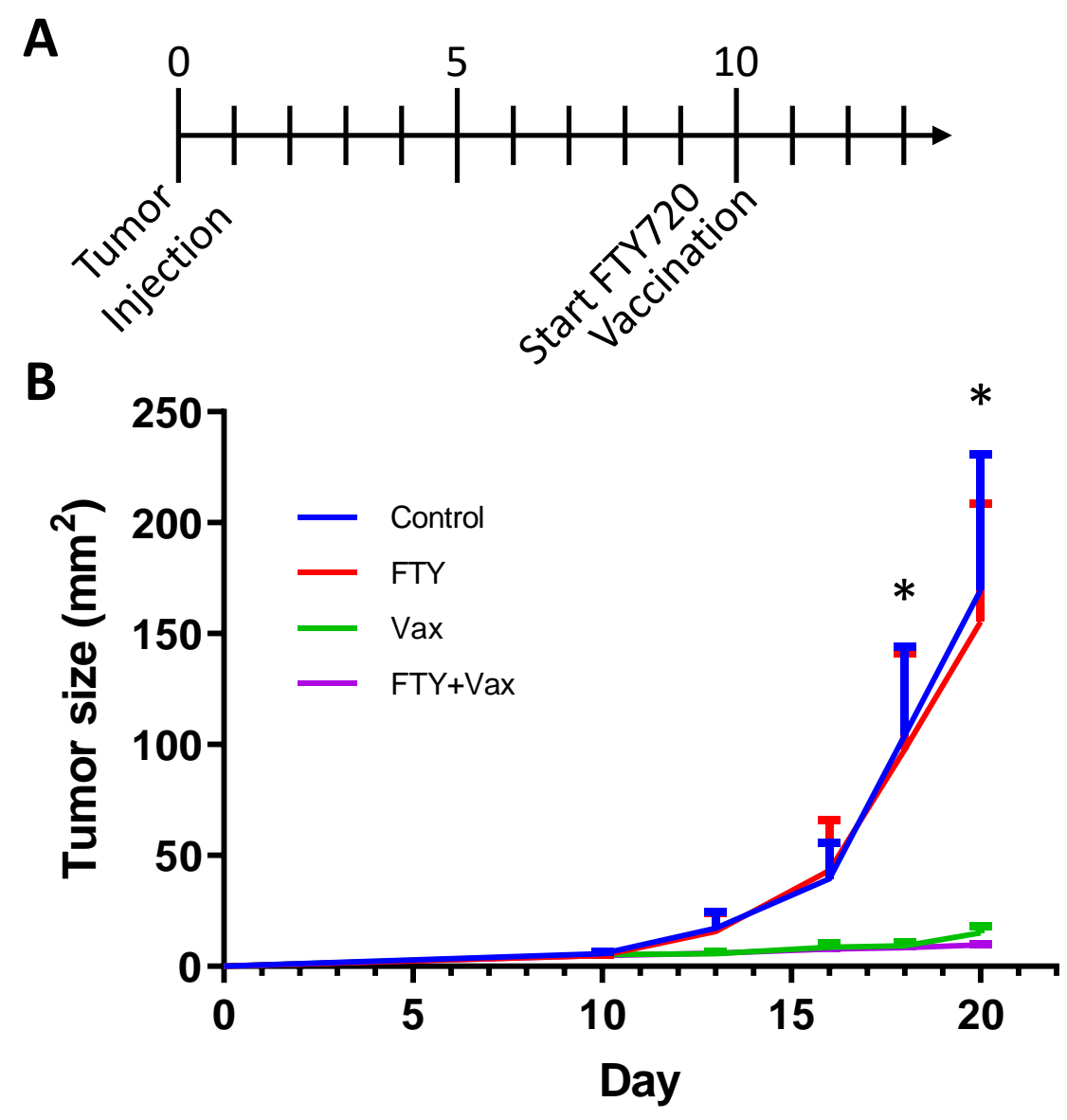
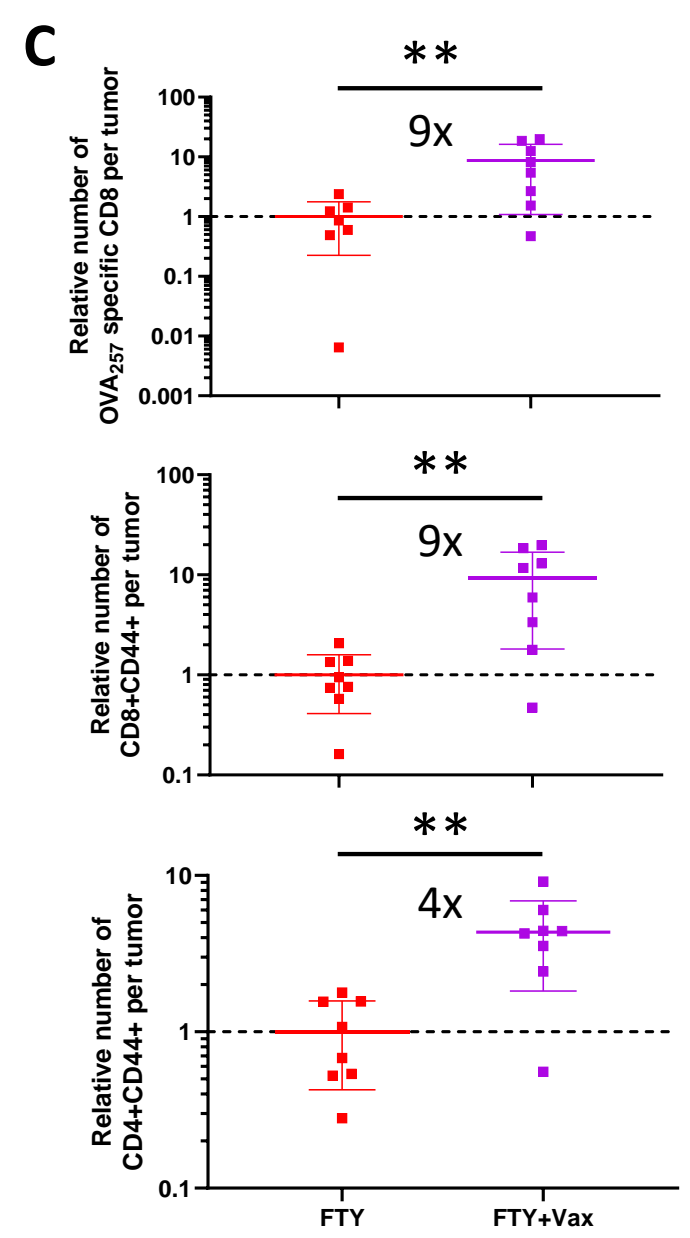
Fig 3
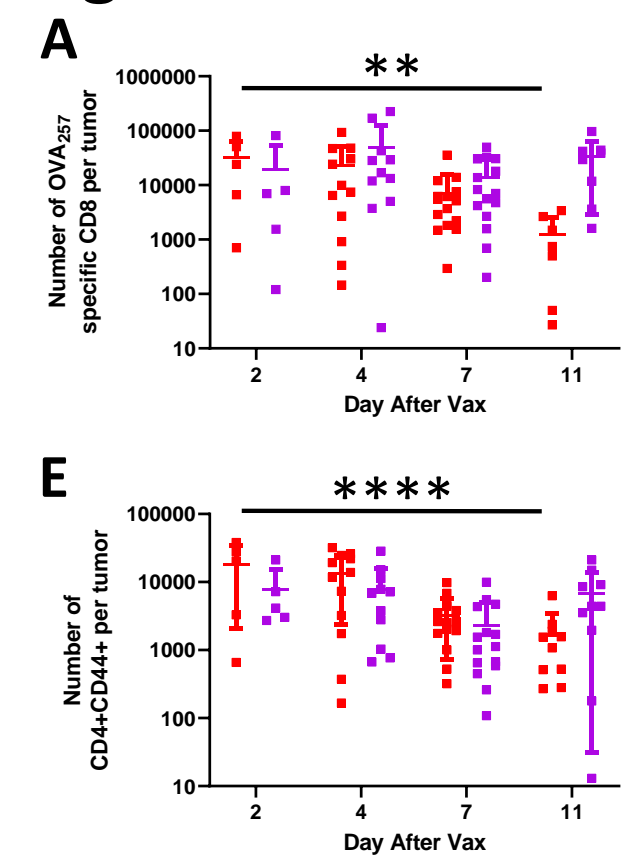

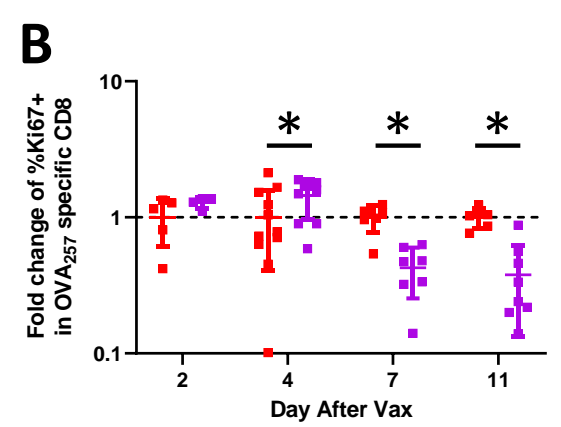

C
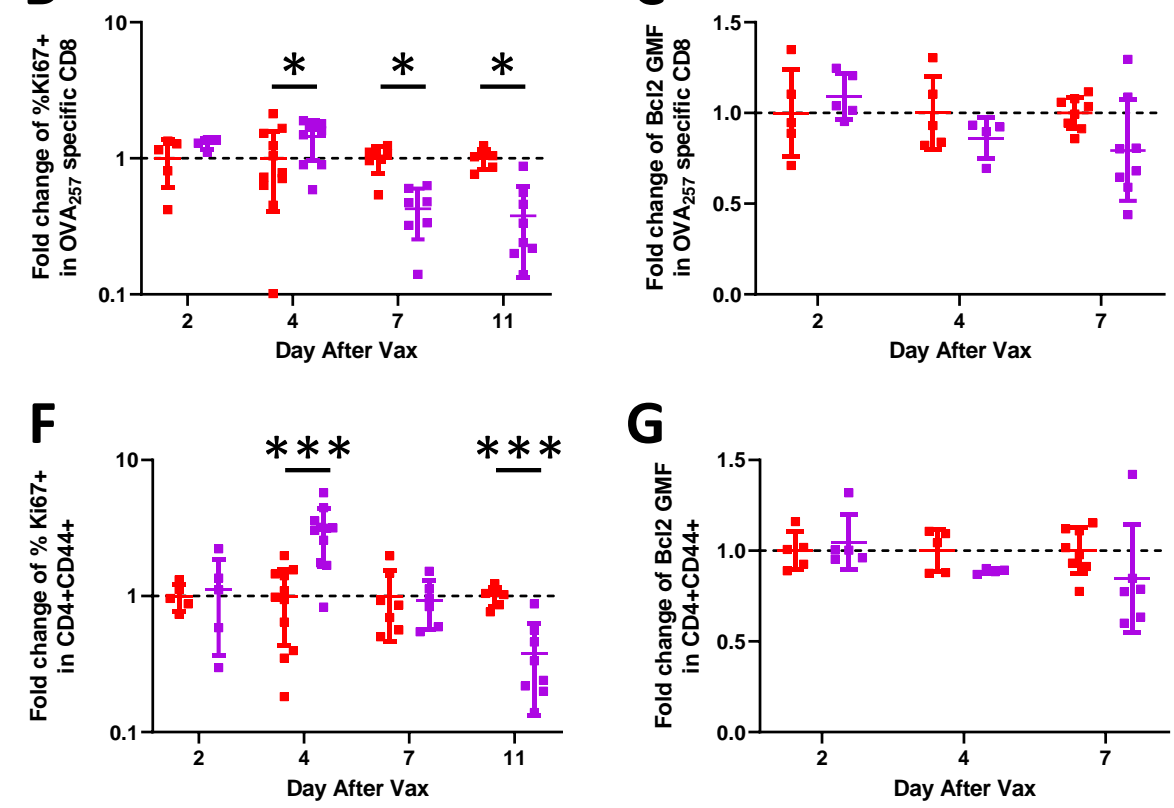

G

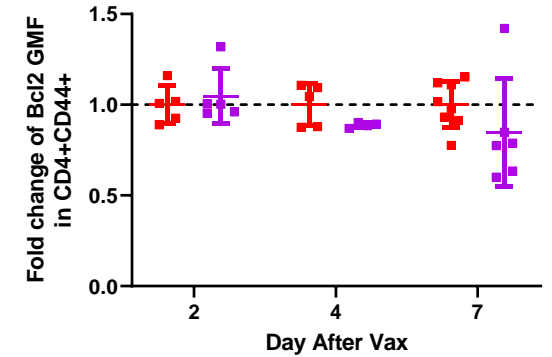

- FTY

- FTY+Vax
D

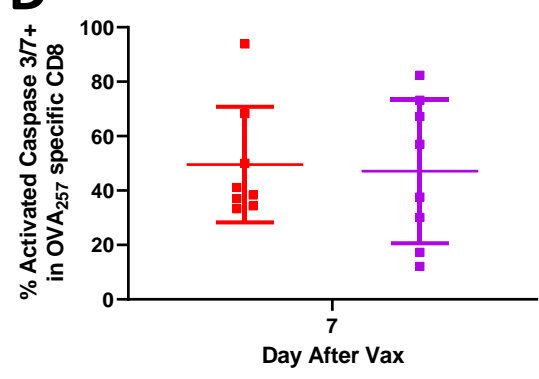

H

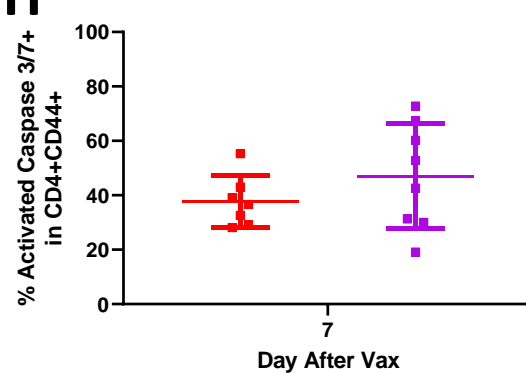


Fig 4

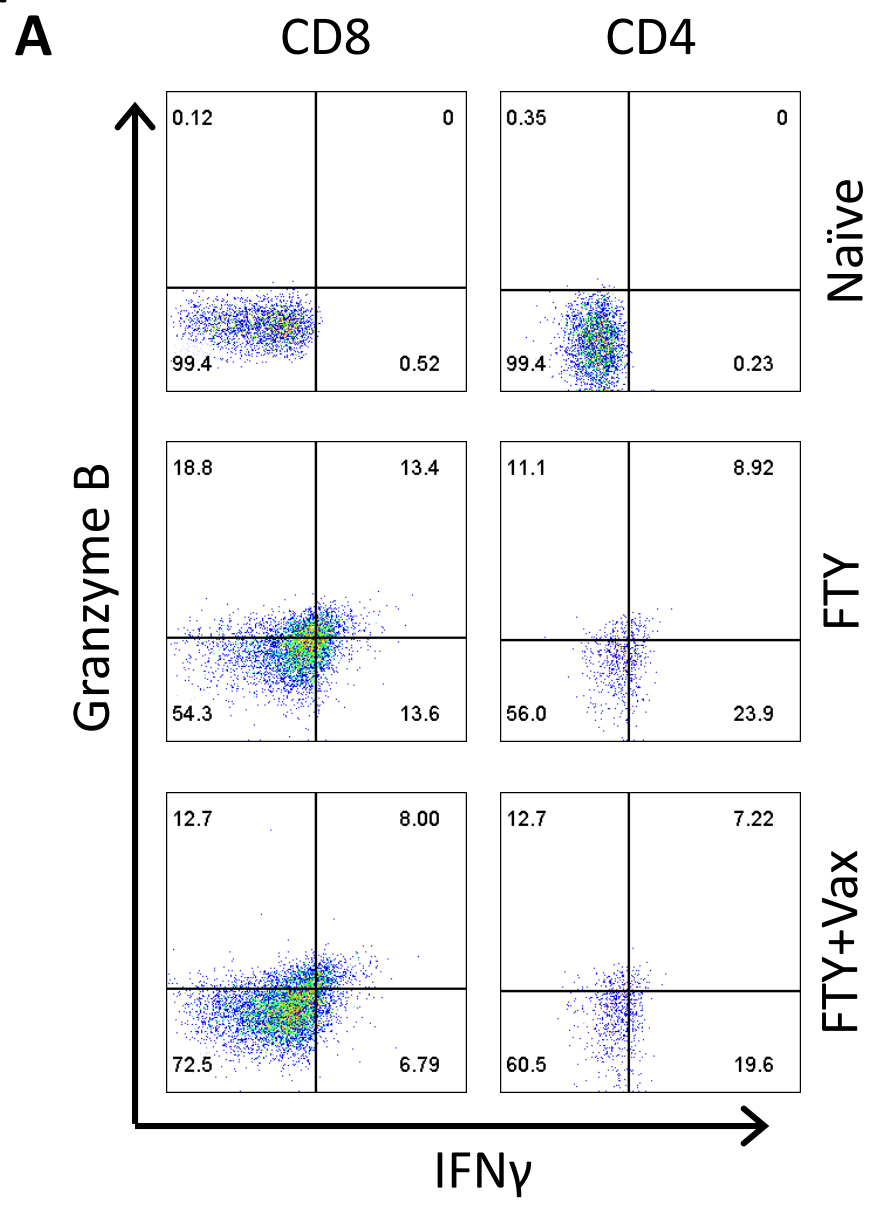

B

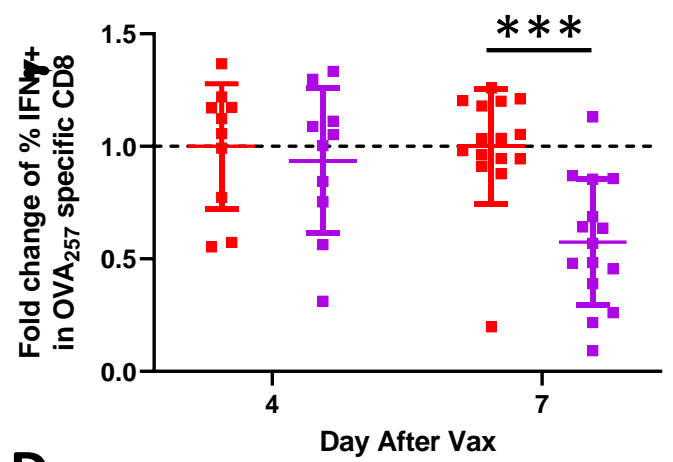

D

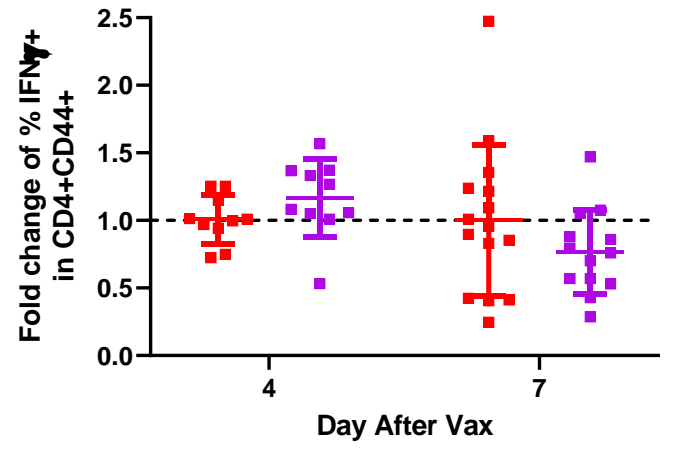

C

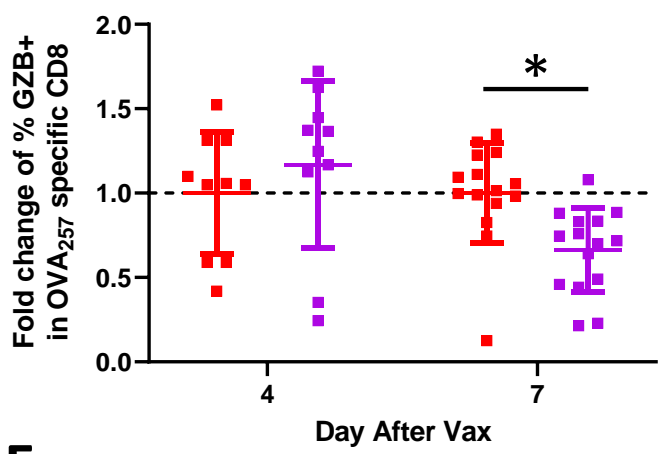

E

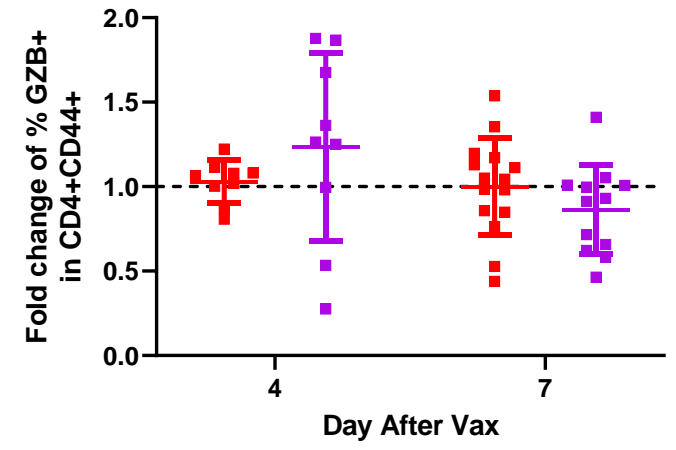


Fig 5
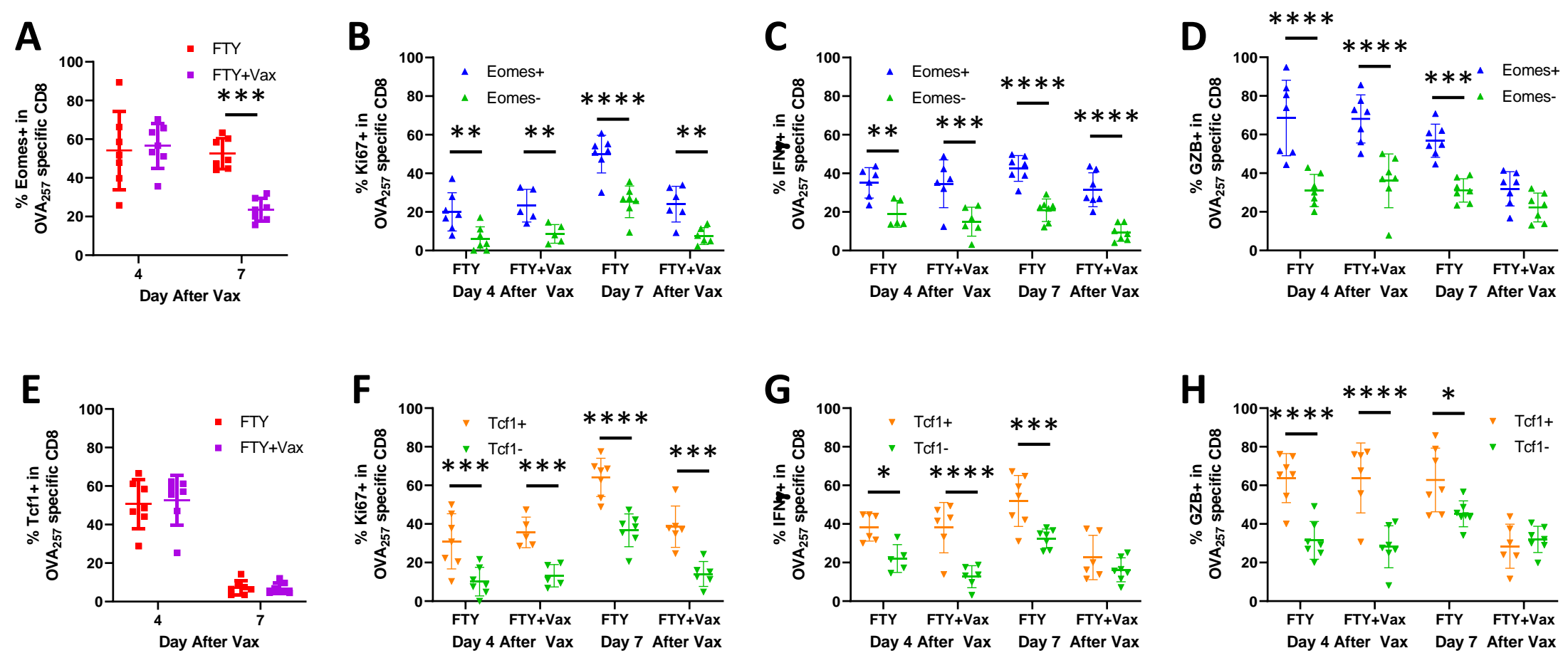
Fig 6

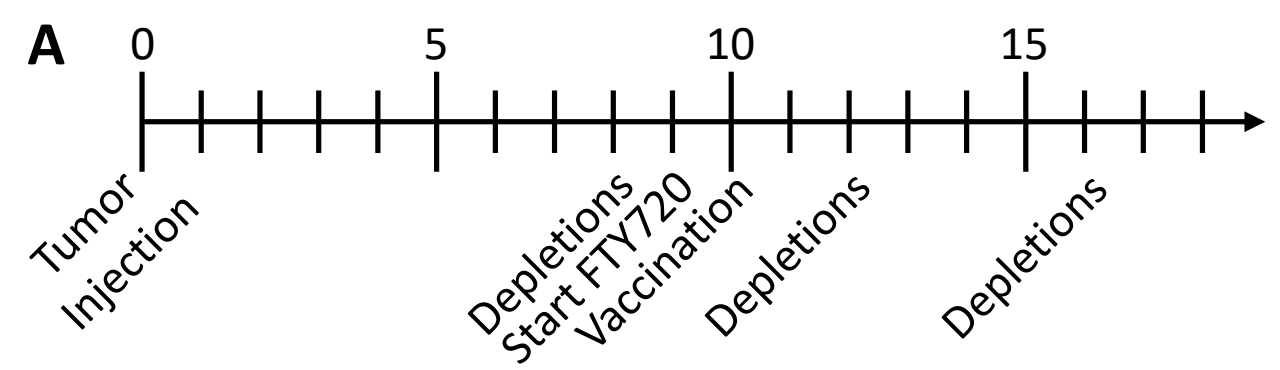

B

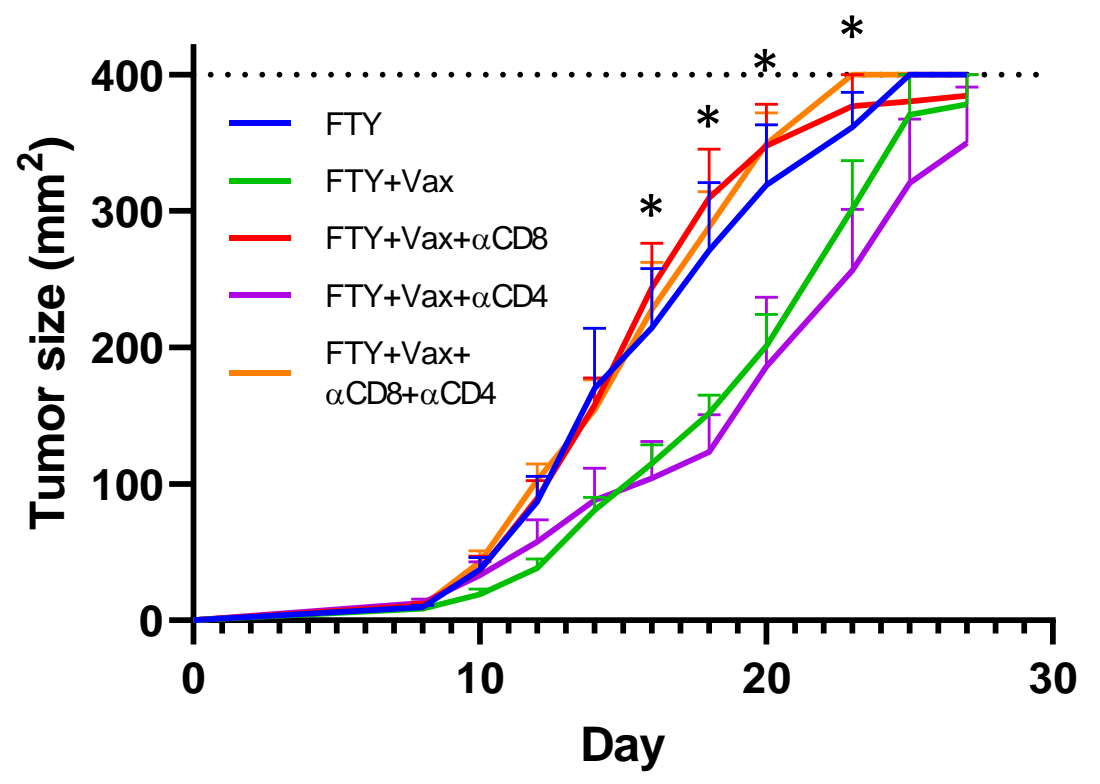

